\title{
Subjective and Objective Effects of Tablet's Pixel Density
}

\author{
Lars Lischke, Sven Mayer, Katrin Wolf, Alireza Sahami, Niels Henze \\ VIS, University of Stuttgart \\ Stuttgart, Germany \\ \{firstname.lastname\}@ vis.uni-stuttgart.de
}

\begin{abstract}
Pixel densities are increasing rapidly. We can observe this trend in particular for mobile devices like smartphones and tablets. Previous work revealed an effect of pixel density on subjective feedback and objective performance only for low resolution cathode ray tube screens. It is unclear if this effect persists for the four times higher pixel densities of current mobile devices. Therefore, we conducted a study to compare four pixel densities with $359,180,120$, and 90 pixels per inch. While participants performed three tasks involving images, text and videos on a tablet, we measured perceived effort, perceived visual quality, task completion time, error rate, and body pose. Our results show that the effect of the pixel density highly depends on the content. We found that only for text, the four pixel densities have clearly different perceived media qualities. Pixel density seems to have a smaller effect on perceived media quality for images and videos and we found no effect on objective measures. Results show that text should be displayed in high resolution, while this is less important for images and videos.
\end{abstract}

\section{Author Keywords}

Pixel density; Resolution; Tablet;

\section{ACM Classification Keywords}

H.5.m. Information Interfaces and Presentation (e.g. HCI): Miscellaneous

\section{INTRODUCTION \& BACKGROUND}

The resolution of computer displays constantly increased over the last decades. While the highest resolution of the first IBM PC introduced in 1981 was $640 \times 200$ pixels, current desktop screens have often a full high definition resolutions of $1980 \times 1080$ pixels or higher. Similar trends can be observed in other domains. In particular, the screen resolution of mobile devices increased. While the first Apple iPhone, introduced in 2007, and the first Android device, introduced in 2008 , had a resolution of $320 \times 480$ pixels, recent devices, such as the Samsung Galaxy S5 come with $1920 \times 1080$ pixels. Although the screen size has been increased at the same

Paste the appropriate copyright statement here. ACM now supports three different copyright statements:

- ACM copyright: ACM holds the copyright on the work. This is the historical approach.

- License: The author(s) retain copyright, but ACM receives an exclusive publication license.

- Open Access: The author(s) wish to pay for the work to be open access. The additional fee must be paid to ACM.

This text field is large enough to hold the appropriate release statement assuming it is single spaced. time, the resolution has grown faster. This results in an increase of the typical pixel density, measured in pixels per inch (PPI), from 170 PPI in 2007 up to 432 PPI in 2014.

Previous research investigated the effect of visual displays. In particular, researchers looked at the difference between reading on papers and on computer screens. In contrast to earlier work [4], results by Ball and Hourcade show that comprehension and reading speed is similar using paper and computer displays [2]. The authors explain the contradiction of previous work with participants' increased familiarity but also through the improvement of technology. Thus, the work suggests that the characteristics of the computer screen have an effect on real world tasks. Menozzi et al. compared different display technologies using a visual search task [6]. While the results show that improved output devices can increase users' performance they also suggest that even clear differences do not necessarily result in a measurable effect.

Surprisingly only few researchers investigated the effect of resolution or pixel density. Ziefle examined the effect of cathode ray tube (CRT) screens' pixel density (60 PPI and $120 \mathrm{PPI}$ ) in comparison to text printed with 255 dots per inch (DPI) [10]. For a proof-reading task, she found that paper significantly outperformed the other conditions but observed no significant difference between the two screen conditions. In a complementing study, however, Ziefle compared three pixel densities (62 PPI, 69 PPI, and 89 PPI) and found a difference between the two lower densities and the higher one in terms of subjective feedback and objective performance when searching for characters. When liquid-crystal displays (LCDs) replaced CRTs, studying the effect of pixel density became challenging as one LCD cannot display different densities. Therefore, studies that aimed to compare different densities have been conducted using different displays (e.g. [5, 9]) or with one display by changing the size of the visual output [3]. In both cases, one cannot differentiate between the effect of the display or its size and the effect of the pixel density.

Overall, previous work showed that an increase of pixel density from 69 to 89 PPI can improve participants' subjective ratings and objective performance for one specific task [10]. The pixel density of current devices is, however, much higher than what could be considered by Ziefle at that time. In particular, mobile devices have a pixel density that is almost five times higher than 89 PPI. It remained unclear if further increasing the pixel density from 89 PPI will sustain the positive effect. Furthermore, previous work only found an effect for search tasks using texts. While current mobile devices are 


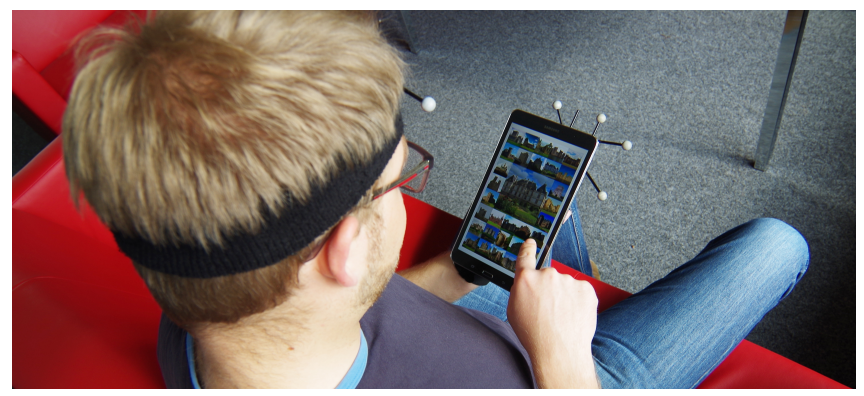

Figure 1. A participant performing the image task.

certainly used for reading, they are also used for a wide range of multimedia tasks involving images and videos [7].

As previous work does not provide insights about the effect of even moderately high pixel densities, it remains unclear if the ongoing increase of density provides an advantage for users. Therefore, we investigate the effect of pixel densities for three tasks using texts, images, and videos. Using a high resolution tablet device with a pixel density of 359 PPI we simulate four lower pixel densities and can thereby investigate different densities using a one device. In the conducted study we assess the effect of the four pixel densities on subjective measures, objective measures, and the participants' pose.

\section{EXPERIMENT}

In this study we used three tasks to assess the effect of pixel density on tablets. We used a tablet device with a resolution of $2560 \times 1600$ pixels and a density of 359 PPI. We combine groups of 4,9 , and 16 pixels resulting in additional pixel densities of $179.5,119.7$, and 89.8 PPI. In the following we use 180, 120 and 90 PPI to describe the densities we used. These three lower pixel densities and the native pixel density result in four conditions. Using a repeated measures design, every participant performed all three tasks with all four conditions. The order of the conditions was balanced using Latin square. We recruited 16 participants ( 5 female) with an age between 21 and $43(M=26.7, S D=5.2)$ through our university's mailing list. All of them had normal or corrected to normal vision.

\section{Tasks}

We designed three tasks which represent actions commonly performed on tablets. Messaging, information look-up and browsing social media are key activities performed on tablets [7]. On a more abstract level looking at and comparing images, reading texts, and watching videos are sub-tasks of these tasks.

In the image comparison task we presented five sets of 32 images. Every image set contained images of persons or objects. All images were collected by searching for keywords on Flickr. As categories we used portraits of women and men, landscape photos of castles, cars, and coins. All images in one set were comparable in terms of color and perspective. Thereby we tried to minimize color-based search strategies. The images were arranged in a grid with five columns and eight rows. All images had a size of $21.2 \mathrm{~mm} \times 21.2 \mathrm{~mm}$
$(300 \times 300$ pixels on the highest density). In the upper center we placed one image which was three times lager $(63.8 \mathrm{~mm} \times 63.8 \mathrm{~mm})$. One of the smaller images showed the same person or object as the larger one but from a different perspective. Participants' task was to compare the small images to the larger image and to select the matching image as fast as possible. Figure 1 shows the task with a participant searching the correct image. Every participant performed the task using each of the five categories and each of the four pixel density. We ensured that every image was only shown once to an individual participant. Furthermore the positions of the small images were randomized. We will call this "image task".

The second task was to count how often a specific word appears in a given text. Before one text was shown to the user we displayed a message that specified the word that should be counted. To support the participant the target word was displayed in in the upper left corner while the text was shown. We selected 12 text sequences with a length of 300 words from different fairy tales in English language. All words which should be counted were common English words, like "she", "with" or "into" with a length of three or four characters. The target word was included in the text between one and eight times. As font we used a regular font (Sans) without serif with a font size of 45 pixels at full resolution. This equals a height of $3.2 \mathrm{~mm}$. The font style and size is comparable to the standard font on the used tablet. We placed the text in the center of the screen. On the bottom of the screen we placed ten buttons to enter how often the target word was counted in the text. For every condition we repeated this task three times. In the following we call this task "text task".

In the last task the participant watched three videos with a length of 10 seconds that showed five people standing in a circle and passing three balls around. The position of the camera was fixed and showed the whole group all the time. The task for the participants was to count the total number of throws in the video. We decided to use videos in which people are passing balls around, because the action happening in all videos are comparable. Each video was played once with a frame rate of $20 \mathrm{fps}$. Participants could not pause or replay the video. After watching a video, participants were asked to enter the number of throws. We call this "video task".

\section{Procedure}

To measure the distance between the tablet and the eyes of the participant while he or she was performing the task, we used a motion capture system. Therefore we mounted optical markers on the tablet and asked the participants to wear a headband with optical markers. While the participant was performing the tasks he or she was sitting on a chair without using a table. Every participant performed 33 trails which results from four pixel densities times the five sets of images, the three sets of texts and the three sets of videos. After performing one task on one density, we asked to answer two questions. First, to rate perceived mental effort (PE) on SMEQ scale [11]. This scale goes from 0 (no effort) to 150 (high effort) and is known to be very sensitive with small sample sizes [8]. Second, to rate perceived media quality (PMQ) on ITU-T P.910 ques- 


\begin{tabular}{|c|c|c|c|c|c|c|c|c|c|}
\hline & & \multicolumn{2}{|c|}{90 PPI } & \multicolumn{2}{|c|}{120 PPI } & \multicolumn{2}{|c|}{180 PPI } & \multicolumn{2}{|c|}{359 PPI } \\
\hline & & M & $S D$ & $M$ & $S D$ & $M$ & $S D$ & $M$ & $S D$ \\
\hline \multirow{3}{*}{$\frac{\sqrt[y]{\tilde{E}}}{\tilde{E}}$} & Image & 4.01 & 2.40 & 4.78 & 2.39 & 6.71 & 1.97 & 7.36 & 1.44 \\
\hline & Text* & 2.15 & 1.58 & 3.95 & 1.71 & 6.03 & 2.00 & 8.05 & 1.40 \\
\hline & Video & 3.72 & 1.86 & 5.16 & 1.56 & 6.59 & 1.57 & 6.14 & 1.80 \\
\hline
\end{tabular}

Table 1. Perceived mental effort $P E$ to perform the task per screen resolution.

* denotes significant effects at the .05 level.

tionnaire, which goes from 0 (bad) to 10 (excellent) [1]. The study tool automatically logged task completion time (TCT) and error rate (ER).

\section{Apparatus}

Differing characteristics of even similar displays, such as contrast, brightness or response time might influence the results of the study. Therefore, we decided to use only one device to minimize the number of uncontrolled variables. In the study we used a regular Android tablet, namely a Samsung Galaxy Tab Pro $8.4^{1}$. The tablet has a 8.4 inches large display with WQXGA $(2560 \times 1600)$ screen resolution. To lower the perceived pixel density we scaled down the material used in the study to the respective density. We scaled the content up again using nearest-neighbor interpolation. Thereby, groups of pixels are combined without any smoothing. With the use of a motion capture system and the attached markers we measured the distance between the eyes of the participant and the tablet, while he or she was performing the tasks.

\section{RESULTS}

For each task, we compared PE, PMQ, TCT, and ER between the four different pixel densities by conducting oneway ANOVAs. Degrees of freedom have been corrected using Greenhouse-Geisser estimation of sphericity if necessary.

As post-hoc tests, we conducted t-tests with Bonferroni correction. Table 2 shows the resulting values for PMQ per pixel density and task and Table 1 shows the resulting values for PE per pixel density and task. Overall, we found no significant effects on distance between tablet and head and on error rate.

\section{Image Task}

We could not show a significant effect of pixel density on PE $\left(F_{1.63,24.39}=3.35, p=.061, \eta^{2}=.182\right)$. However, the analysis revealed a significant effect of pixel density on PMQ $\left(F_{3,45}=12.6, p<.001, \eta^{2}=.456\right)$. Post-hoc tests revealed that PMQ for 359 PPI was significantly higher than for 120 PPI $(p<.001)$ as well as than for 90 PPI $(p<.001)$. Furthermore, we found a significant difference on PMQ between 180 PPI and 90 PPI $(p<.015)$. Post-hoc tests revealed no significant difference for all other pairwise comparisons. The analysis revealed significant differences for TCT $\left(F_{3,36}=6.625, p<.003, \eta^{2}=.356\right)$. Post-hoc test revealed significant effects for TCT while performing the task with the highest pixel density (359 PPI) vs.

\footnotetext{
www.samsung.com/us/mobile/galaxy-tab/

SM-T320NZKAXAR (last access 09-19-2014)
}

\begin{tabular}{|c|c|c|c|c|c|c|c|}
\hline \multicolumn{2}{|c|}{90 PPI } & \multicolumn{2}{|c|}{120 PPI } & \multicolumn{2}{|c|}{180 PPI } & \multicolumn{2}{|c|}{359 PPI } \\
\hline$M$ & $S D$ & $M$ & $S D$ & $M$ & $S D$ & $M$ & $S D$ \\
\hline
\end{tabular}

$\begin{array}{lllllll}\text { Image*37.38 }^{*} 21.69 & 55.94 & 27.98 & 42.25 & 35.82 & 39.50 & 27.91\end{array}$

$\stackrel{\frac{y}{5}}{\stackrel{\Xi}{\leftrightarrows}}$

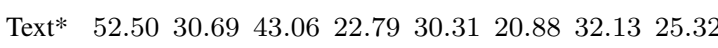

Video* 41.8831 .0235 .5623 .4131 .8124 .7128 .1322 .01

Table 2. Perceived media quality PMQ per screen resolution.

* denotes significant effects at the .05 level.

120 PPI $(p<.045)$. Furthermore, the differences between 120 PPI and 90 PPI were significant $(p<.005)$. However, the analysis could not reveal significant differences between the other pixel densities.

\section{Text task}

Significant effects for PE were found $\left(F_{3,45}=13.13\right.$, $\left.p<.001, \eta^{2}=.467\right)$. Post-hoc test showed that PE for performing the task at 359 PPI was significantly lower than at 90 PPI $(p<.009)$. Furthermore, the analysis reveals significant differences between 180 PPI and 120 PPI $(p<.018)$ and between 180 PPI and 90 PPI $(p<.002)$. However the analysis revealed no significant effect between all other resolutions. The analysis revealed significant differences on PMQ $\left(F_{3,45}=48.52, p<.001, \eta^{2}=.764\right)$. Posthoc tests showed that all used pixel densities were perceived significantly different (359 PPI vs. 180 PPI: $p<.027$; 180 PPI vs. 120 PPI: $p<.003$, and all other pairs: $p<.001$. The analysis of TCT revealed no significant differences $\left(F_{3,36}=1.728, p=.179, \eta^{2}=.126\right)$.

\section{Video task}

The analysis revealed no significant effects on PE $\left(F_{3,45}=3.82, p=.016, \eta^{2}=.203\right)$. However, the analysis revealed significant differences for PMQ $\left(F_{3,45}=18.38\right.$, $\left.p<.001, \eta^{2}=.551\right)$. Post-hoc tests showed that PMQ differs significantly between the highest (359 PPI) and the lowest (90 PPI) pixel density $(p<.003)$. Furthermore, the three lower pixel densities were perceived significantly different (180 PPI vs. 120 PPI: $p<.009$; 180 PPI vs. 90 PPI: $p<.001 ; 120$ PPI vs. 90 PPI: $p<.008)$. The analysis of TCT revealed no significant differences $\left(F_{3,36}=1.012\right.$, $\left.p=.399, \eta^{2}=.078\right)$.

\section{DISCUSSION \& CONCLUSION}

The results of our study show that the influence of pixel density depends on the performed task. Perceived media quality differs for all three tasks. The results suggest that pixel density is particularly important for text related tasks. Only for the text task, participants rated all four presented pixel densities significantly different. In addition, perceived mental effort while performing the text related task differs significantly between the different pixel densities. This indicates that a higher pixel density and thereby a clearer font makes reading easier. In contrast, not all densities in the image task were rated significantly different. We found no differences between 359 PPI and 180 PPI, 180 PPI and 120 PPI, and between 120 PPI and 90 PPI. This indicates that the perceived differences of the visual quality for images did not vary as 
much as for text. Perceived video quality differs between pixel densities of 180 PPI, 120 PPI, and 90 PPI. However, perceived quality of the highest pixel density is only different compared to the lowest quality. This indicates that for video watching a higher quality might not be necessary. Overall, we could not show that a higher pixel density is beneficial in terms of task completion time or error rate. Also the results demonstrate that the distance between users' head and device does not change depending on the pixel density of the screen.

In contrast to previous work [10] that investigated the effect of pixel density, our work focuses on mobile devices which today have screens with a much higher pixel density. Moreover, we use additional types of content, which are today commonly used on smartphones or tablets. As Ziefle's work [10] indicates, the effect of pixel density is task dependent. In contrast to Ziefle's work on lower pixel densities [10], our results do not show a continuously increasing positive effect of the display's pixel density on TCT and ER. This might have different reasons. First, low visual quality might be compensated by the human vision. Second, Menozzi et al. [6] indicate that obvious differences in the visual quality do not necessarily haven an objective effect.

Our results suggest that pixel densities, used for modern smartphones and tablets, have little positive effect on objective measurable variables. However, pixel density has a clear effect on perceived visual quality which is leveling off for high densities. Hence the results rise the question, if a further increase of screen resolution is necessary. In particular for price sensitive and emerging markets economically priced hardware with an screen resolution of 180 PPI could be sufficient. The results indicate that reading on a screen with a resolution of $180 \mathrm{PPI}$ has the lowest PE. If we consider only measurable performance as important, even cheaper hardware is suitable. Additionally the perceived visual quality depends on the media type. While visual text quality is very distinguishable, the pixel density is less important for videos. This shows even more potential for using economically priced hardware. The visual quality of videos seems to be less important. This allow to deploy less computational power to display videos in devices for emerging markets. This affects also the needed quality of network connections, because it might be not necessary to send large amount of data to display high resolution videos. The perceivable differences in the media quality allow in contrast also to argue for costly high quality displays. This discussion is in particular important, if we transfer our results from tablets to other mobile devices, like smartwatches. Smartwatches are designed to provide fast access to bits of information. Our results show that this is still possible without measurable disadvantages on displays with very low resolution. The screen resolution has the largest perceivable effect on reading. However, smartwatches are not designed to read longer text. Hence, we could argue that lower screen resolutions are reasonable for smartwatches. This could allow to use less computational power and thereby save battery life.

Our work raises different related research questions. The influence of the screen size is strongly connected to effects we analyzed. The screen size can influence the pixel density and the amount of content which can be displayed at once. In this study we compared only different screen resolutions on the same screen size. Furthermore the size of the content, e.g. the size of the text font, might interfere with the pixel density and thereby effect user's performance and the task load. In this initial study we used content size which is typical for this tablet. The question about influence of the screen size, calls for analyzing same variables in other settings. So it would be interesting to proof our assumption that high display resolutions are not necessary on smartwatches. To analyze the effect of display resolution in a desktop settings would be interesting, because the user is using the screen often a longer time.

Acknowledgments: This work is partly financial supported by German Research Foundation (DFG) within Cluster of Excellence in Simulation Technology (EXC 310/2) at the University of Stuttgart.

\section{REFERENCES}

1. Recommendation ITU-T P.910 Subjective video quality assessment methods for multimedia applications. Telecom. Standard. Sector of ITU (2008).

2. Ball, R., and Hourcade, J. P. Rethinking reading for age from paper and computers. IJHCI 27, 11 (2011).

3. Chen, C.-H., and Chiang, S.-Y. Effects of screen resolution and column ratio on search performance and subjective preferences. Displays 33, 1 (2012).

4. Dillon, A. Reading from paper versus screens: A critical review of the empirical literature. Ergonomics 35, 10 (1992).

5. Huang, D.-L., Patrick Rau, P.-L., and Liu, Y. Effects of font size, display resolution and task type on reading chinese fonts from mobile devices. International Journal of Industrial Ergonomics 39, 1 (2009).

6. Menozzi, M., Lang, F., Naepflin, U., Zeller, C., and Krueger, H. Crt versus lcd: Effects of refresh rate, display technology and background luminance in visual performance. Displays 22, 3 (2001).

7. Müller, H., Gove, J., and Webb, J. Understanding tablet use. In Proceedings of MobileHCI (2012).

8. Sauro, J., and Dumas, J. S. Comparison of three one-question, post-task usability questionnaires. In Proceedings of CHI (2009).

9. Yang, S.-N., Tai, Y.-C., Hayes, J. R., and Sheedy, J. Superior smartphone display quality enhances viewing performance and comfort. Faculty Scholarship (2011).

10. Ziefle, M. Effects of display resolution on visual performance. Journal of the Human Factors and Ergonomics Society 40, 4 (1998).

11. Zijlstra, F., and Doorn, L. v. The construction of a scale to measure subjective effort. Tech. Rep. Delft University of Technology (1985). 